\title{
Automatic radiometric normalization of multitemporal satellite imagery
}

\author{
Morton J. Canty ${ }^{\mathrm{a}, *}$, Allan A. Nielsen ${ }^{\mathrm{b}}$, Michael Schmidt ${ }^{\mathrm{c}}$ \\ a Systems Analysis and Technology Evaluation, Jülich Research Center, D-52425 Jülich, Germany \\ ${ }^{\mathrm{b}}$ Informatics and Mathematical Modelling, Technical University of Denmark, DK-2800 Kgs. Lyngby, Denmark \\ ${ }^{\mathrm{c}}$ Department of Geography, University of Bonn, Meckenheimer Allee 166, D-53115 Bonn, Germany
}

Received 24 September 2002; received in revised form 20 March 2003; accepted 13 October 2003

\begin{abstract}
The linear scale invariance of the multivariate alteration detection (MAD) transformation is used to obtain invariant pixels for automatic relative radiometric normalization of time series of multispectral data. Normalization by means of ordinary least squares regression method is compared with normalization using orthogonal regression. The procedure is applied to Landsat TM images over Nevada, Landsat ETM+ images over Morocco, and SPOT HRV images over Kenya. Results from this new automatic, combined MAD/orthogonal regression method, based on statistical analysis of test pixels not used in the actual normalization, compare favorably with results from normalization from manually obtained time-invariant features.
\end{abstract}

(C) 2004 Elsevier Inc. All rights reserved.

Keywords: MAD transformation; Orthogonal regression; Radiometric normalization

\section{Introduction}

Radiometric normalization of satellite imagery requires, among other things, an atmospheric correction algorithm and the associated atmospheric properties at the times of image acquisition. For most historical satellite scenes, such data are not available and even for planned acquisitions they may be difficult to obtain. A relative normalization based on the radiometric information intrinsic to the images themselves is an alternative whenever absolute surface radiances are not required, for example in change detection applications or for supervised land cover classification.

Several methods (Du et al., 2002; Furby \& Campbell, 2001; Hall et al., 1991; Moran et al., 1992; Schott et al., 1988) have been proposed for the relative radiometric normalization of multispectral images taken under different conditions at different times. All proceed under the assumption that the relationship between the at-sensor radiances recorded at two different times from regions of constant reflectance is spatially homogeneous and can be approximated by linear functions. The most difficult and time-

\footnotetext{
* Corresponding author. Tel.: +49-2461-61-4885; fax: +49-2461-612540.

E-mail address: m.canty@fz-juelich.de (M.J. Canty).
}

consuming aspect of all of these methods is the determination of suitable time-invariant features upon which to base the normalization.

Nielsen et al. $(2002,1998)$ recently proposed a change detection technique, called multivariate alteration detection (MAD), which is invariant to linear and affine scaling. Thus, if one uses MAD for change detection applications, preprocessing by linear radiometric normalization is superfluous. However, radiometric normalization of imagery is important for many other applications, such as mosaicking, tracking vegetation indices over time, supervised and unsupervised land cover classification, etc. Furthermore, if some other, non-invariant change detection procedure is preferred, it must generally be preceded by radiometric normalization.

We have applied the MAD transformation to select the no-change pixels in bitemporal images, and then used them for radiometric normalization. The procedure is simple, fast and completely automatic and compares very favorably with normalization using hand-selected, time-invariant features.

\section{Selecting invariant pixels}

In order to mask out the change pixels in a bitemporal scene, we first form linear combinations of the intensities for 
all $N$ channels in the two images, acquired at times $t_{1}$ and $t_{2}$. Representing the intensities by the random vectors $\mathbf{F}$ and $\mathbf{G}$, respectively, we have

$U=\mathbf{a}^{\top} \mathbf{F}=a_{1} F_{1}+a_{2} F_{2}+\ldots+a_{N} F_{N}$

$V=\mathbf{b}^{\top} \mathbf{G}=b_{1} G_{1}+b_{2} G_{2}+\ldots+b_{N} G_{N}$,

where $\mathbf{a}$ and $\mathbf{b}$ are constant vectors. Nielsen et al. suggest determining the transformation coefficients so that the positive correlation between $U$ and $V$ is minimized. This means that the difference image $U-V$ will show maximum spread in its pixel intensities. If we assume that the spread is primarily due to actual changes that have taken place in the scene over the interval $\left[t_{2}, t_{1}\right]$, then this procedure will enhance those changes as much as possible.

Specifically, we seek linear combinations such that

$$
\begin{aligned}
& \operatorname{Var}(U-V)=\operatorname{Var}(U)+\operatorname{Var}(V)-2 \operatorname{Cov}(U, V) \\
& \quad \rightarrow \text { maximum, }
\end{aligned}
$$

subject to the constraints

$\operatorname{Var}(U)=\operatorname{Var}(V)=1$

and with $\operatorname{Cov}(U, V)>0$. Note that under these constraints

$\operatorname{Var}(U-V)=2(1-\rho)$,

where $\rho$ is the correlation of the transformed vectors $U$ and $V$,

$\rho=\operatorname{Corr}(U, V)=\frac{\operatorname{Cov}(U, V)}{\sqrt{\operatorname{Var}(U) \operatorname{Var}(V)}}$

The combined random vector for the bitemporal scene (F G) is assumed to have zero mean and variance-covariance matrix

$$
\left(\begin{array}{ll}
\sum_{f f} & \sum_{f g} \\
\sum_{g f} & \sum_{g g}
\end{array}\right),
$$

so that

$$
\begin{aligned}
\operatorname{Var}(U) & =\mathbf{a}^{\top} \sum_{f f} \mathbf{a}, \operatorname{Var}(V)=\mathbf{b}^{\top} \sum_{g g} \mathbf{b} \text { and } \operatorname{Cov}(U, V) \\
& =\mathbf{a}^{\top} \sum_{f g} \mathbf{b} .
\end{aligned}
$$

Extremalizing the covariance $\operatorname{Cov}(U, V)$ under the constraints (Eq. (2)) is equivalent to extremalizing the unconstrained function

$L=\mathbf{a}^{\top} \sum_{f g} \mathbf{b}-\frac{v}{2}\left(\mathbf{a}^{\top} \sum_{f f} \mathbf{a}-1\right)-\frac{\mu}{2}\left(\mathbf{b}^{\top} \sum_{g g} \mathbf{b}-1\right)$, where $v$ and $\mu$ are Lagrange multipliers. This leads to the coupled generalized eigenvalue problems

$\sum_{f g} \sum_{g g}^{-1} \sum_{g f} \mathbf{a}=\rho^{2} \sum_{f f} \mathbf{a}$

$\sum_{g f} \sum_{f f}^{-1} \sum_{f g} \mathbf{b}=\rho^{2} \sum_{g g} \mathbf{b}$.

Thus, the desired projections $U=\mathbf{a}^{\top} \mathbf{F}$ are given by the eigenvectors $\mathbf{a}_{1} \ldots \mathbf{a}_{N}$ corresponding to the generalized eigenvalues

$\rho_{1}^{2} \geq \ldots \geq \rho_{N}^{2}$

of $\sum_{f g} \sum_{g g}^{-1} \sum_{g f}$ with respect to $\sum_{f f}$. Similarly the desired projections $V=\mathbf{b}^{\top} \mathbf{G}$ are given by the eigenvectors $\mathbf{b}_{1} \ldots \mathbf{b}_{N}$ of $\sum_{g f} \sum_{f f}^{-1} \sum_{f g}$ with respect to $\sum_{g g}$ corresponding to the same eigenvalues. Nielsen et al. (1998) refer to the $N$ difference components

$\mathrm{MAD}_{i}=U_{i}-V_{i}=\mathbf{a}_{i}^{\top} \mathbf{F}-\mathbf{b}_{i}^{\top} \mathbf{G}, \quad i=1 \ldots N$,

as the multivariate alteration detection (MAD) components of the combined bitemporal image. The covariances of the MAD components are given by

$\operatorname{Cov}\left(U_{i}-V_{i}, U_{j}-V_{j}\right)=2 \delta_{i j}\left(1-\rho_{j}\right)$,

where $\delta_{i j}$ is Kronecker's delta,

$\delta_{i j}= \begin{cases}1 & \text { for } i=j \\ 0 & \text { for } i \neq j .\end{cases}$

The components are thus orthogonal (uncorrelated) with variances

$\operatorname{Var}\left(U_{i}-V_{i}\right)=\sigma_{\mathrm{MAD}_{i}}^{2}=2\left(1-\rho_{i}\right)$.

The last MAD component has maximum spread in its pixel intensities and, ideally, maximum change information. The second-to-last component has maximum spread subject to the condition that the pixel intensities are statistically uncorrelated with those in the first MAD component, and so on.

The MAD components are invariant under linear transformations of the original image intensities. We can see this as follows. Suppose the second image $\mathbf{G}$ is transformed according to some linear transformation $\mathbf{H}=\mathbf{T G}$. The relevant covariance matrices are then

$\sum_{f g}^{\prime}=\left\langle\mathbf{F} \mathbf{H}^{\top}\right\rangle=\sum_{f g} \mathbf{T}^{\top}$

$\sum_{g f}^{\prime}=\left\langle\mathbf{H F}^{\top}\right\rangle=\mathbf{T} \sum_{g f}$

$\sum_{f f}^{\prime}=\sum_{f f}$

$\sum_{g g}^{\prime}=\left\langle\mathbf{H} \mathbf{H}^{\top}\right\rangle=\mathbf{T} \sum_{g g} \mathbf{T}^{\top}$. 
The eigenvalue problems (Eq. (4)) are therefore equivalent to

$$
\begin{aligned}
& \sum_{f g} \mathbf{T}^{\top}\left(\mathbf{T} \sum_{g g} \mathbf{T}^{\top}\right)^{-1} \mathbf{T} \sum_{g f} \mathbf{a}=\rho^{2} \sum_{f f} \mathbf{a} \\
& \mathbf{T} \sum_{g f} \sum_{f f}^{-1} \sum_{f g} \mathbf{T}^{\top} \mathbf{c}=\rho^{2} \mathbf{T} \sum_{g g} \mathbf{T}^{\top} \mathbf{c},
\end{aligned}
$$

where $\mathbf{c}$ is the desired projection for $\mathbf{H}$. These in turn are equivalent to

$\sum_{f g} \sum_{g g}^{-1} \sum_{g f} \mathbf{a}=\rho^{2} \sum_{f f} \mathbf{a}$

$\sum_{g f} \sum_{f f}^{-1} \sum_{f g}\left(\mathbf{T}^{\top} \mathbf{c}\right)=\rho^{2} \sum_{g g}\left(\mathbf{T}^{\top} \mathbf{c}\right)$

which are identical to Eq. (4) with $\mathbf{b}=\mathbf{T}^{\top} \mathbf{c}$. Therefore, the MAD components in the transformed situation are

$$
\begin{aligned}
\mathbf{a}_{i}^{\top} \mathbf{F}-\mathbf{c}_{i}^{\top} \mathbf{H} & =\mathbf{a}_{i}^{\top} \mathbf{F}-\mathbf{c}_{i}^{\top} \mathbf{T} \mathbf{G}=\mathbf{a}_{i}^{\top} \mathbf{F}-\left(\mathbf{T}^{\top} \mathbf{c}_{i}\right)^{\top} \mathbf{G} \\
& =\mathbf{a}_{i}^{\top} \mathbf{F}-\mathbf{b}_{i}^{\top} \mathbf{G}
\end{aligned}
$$

as before. Given this scale invariance, we can select for radiometric normalization all pixel coordinates which satisfy

$\sum_{i=1}^{N}\left(\frac{\mathrm{MAD}_{i}}{\sigma_{\mathrm{MAD}_{i}}}\right)^{2}<t$

where $t$ is a decision threshold. Under the hypothesis of no-change, the above sum of squares of standardized

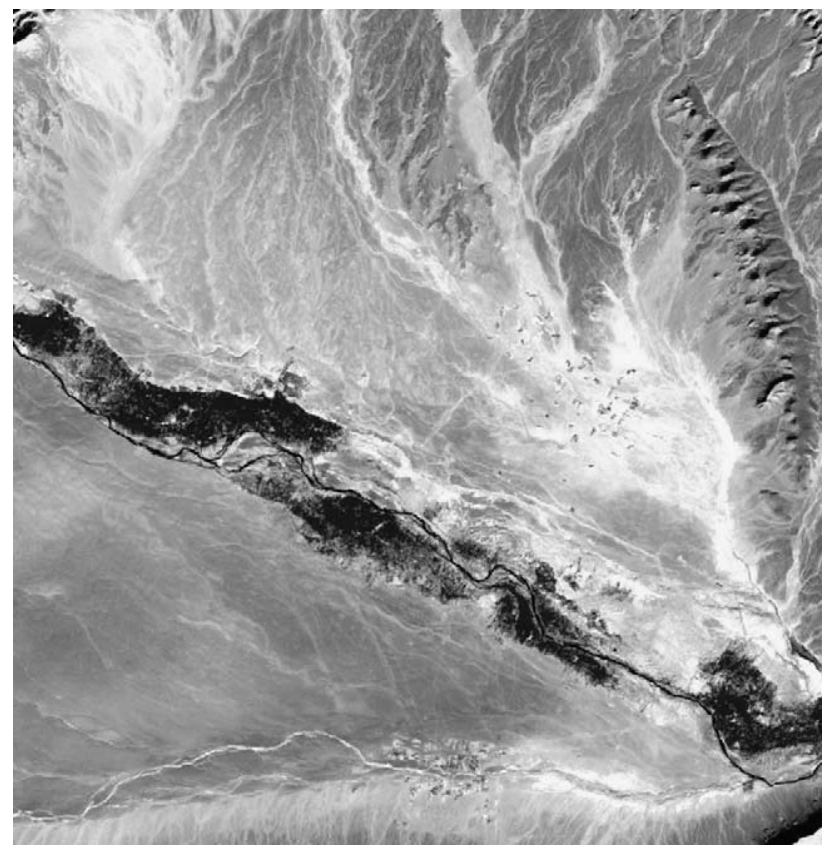

Fig. 1. Landsat-7 ETM+ image from December, 1999 over Morocco.

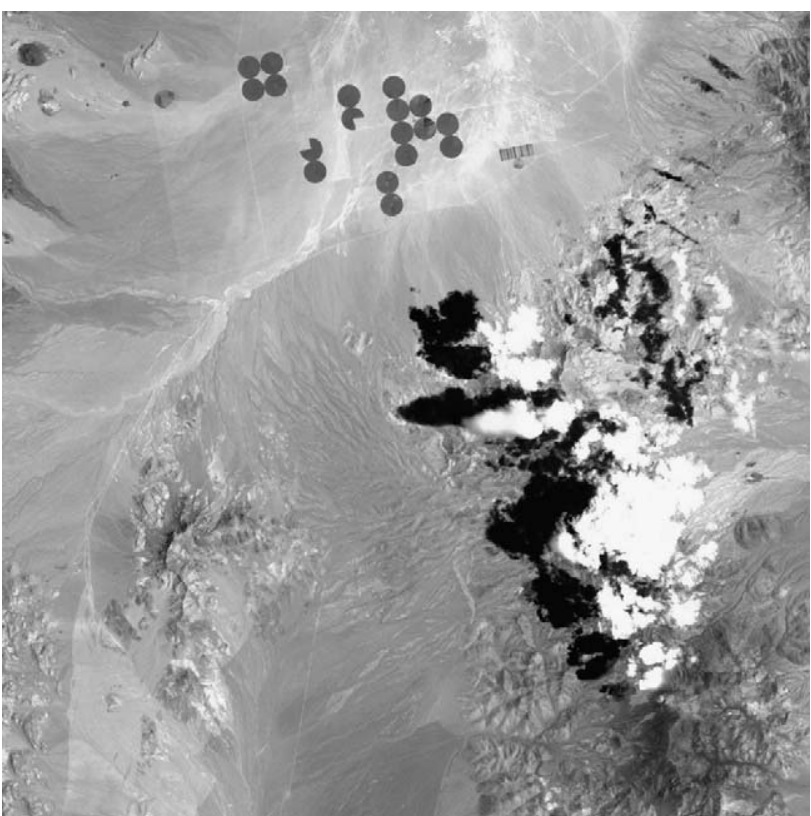

Fig. 2. Landsat-5 TM image from July, 1991 over Nevada.

MAD variables is approximately chi-square distributed with $N$ degrees of freedom. We therefore choose $t=$ $\chi_{N, P=0.01}^{2}$ where $P$ is the probability of observing that value of $t$ or lower.

The pixels thus selected should correspond to truly invariant features so long as the overall radiometric differences between the two images can be attributed to linear effects. Since this method usually identifies quite a large number of no-change pixels, we can, without serious

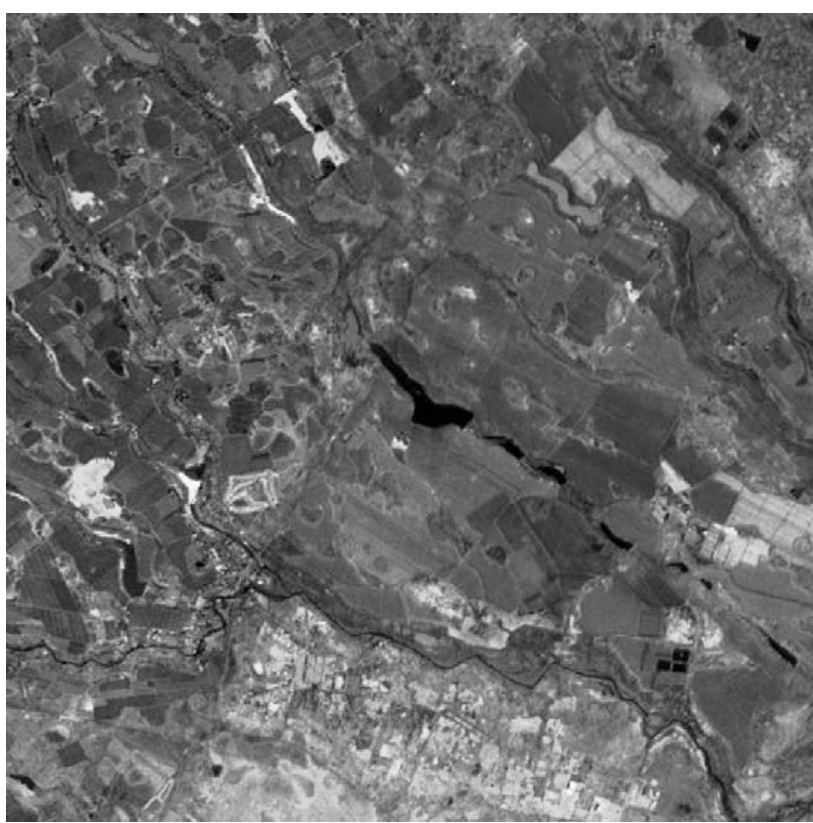

Fig. 3. SPOT HRV image from 1987 over Kenya. 
Table 1

Time-invariant features chosen for normalization to the 1999 scene

\begin{tabular}{lcl}
\hline Feature & Number of pixels & Appearance \\
\hline Clay & 213 & bright \\
Sand & 183 & bright \\
Fixed sand & 9347 & medium \\
Pediment1 & 301 & medium \\
Quarzite & 117 & medium \\
Pediment2 & 365 & dark \\
Dark stones & 233 & dark \\
\hline
\end{tabular}

penalty, reserve some fraction of them for subsequent testing and use the remaining pixels for performing the linear regressions.

With regard to the actual normalization on the basis of the no-change pixels, this is usually done by means of ordinary least squares (OLS) regression analysis, see, e.g. (Yang \& Lo, 2000), which is a method that allows for measurement uncertainty (or error) in one variable only. For radiometric normalization, both variables involved have measurement uncertainty associated with them-in fact which variable is termed reference and which is termed unnormalized data is arbitrary. We have therefore also investigated orthogonal linear regression to perform the actual normalization, as this method treats the data
Table 2

Ordinary least squares regression on manually selected training pixels for the Morocco scenes; $\hat{\alpha}$ is the fitted intercept, $\hat{\beta}$ is the fitted slope, $r$ is the correlation and RMSE is the root mean square error

\begin{tabular}{lllllll}
\hline Band & $\hat{\alpha}$ & $\hat{\sigma}_{\alpha}$ & $\hat{\beta}$ & $\hat{\sigma}_{\beta}$ & $r$ & RMSE \\
\hline 1 & 8.60 & 0.39 & 1.081 & 0.006 & 0.818 & 2.019 \\
2 & -3.00 & 0.24 & 1.184 & 0.004 & 0.928 & 1.845 \\
3 & -7.09 & 0.23 & 1.198 & 0.003 & 0.947 & 2.761 \\
4 & -6.37 & 0.18 & 1.258 & 0.003 & 0.961 & 2.020 \\
5 & 4.76 & 0.23 & 1.081 & 0.003 & 0.927 & 2.891 \\
7 & 5.31 & 0.24 & 1.077 & 0.003 & 0.910 & 2.870 \\
\hline
\end{tabular}

symmetrically. The method is explained in detail in Appendix A.

\section{Data and results}

The data set used to investigate radiometric normalization consisted of Landsat TM (thematic mapper) images over Morocco and Nevada and SPOT HRV (high resolution visible) images over Kenya.

Two Landsat-7 ETM+ (extended thematic mapper) images acquired over Morocco on December 19, 1999 and October 18, 2000 (see Fig. 1) were examined for
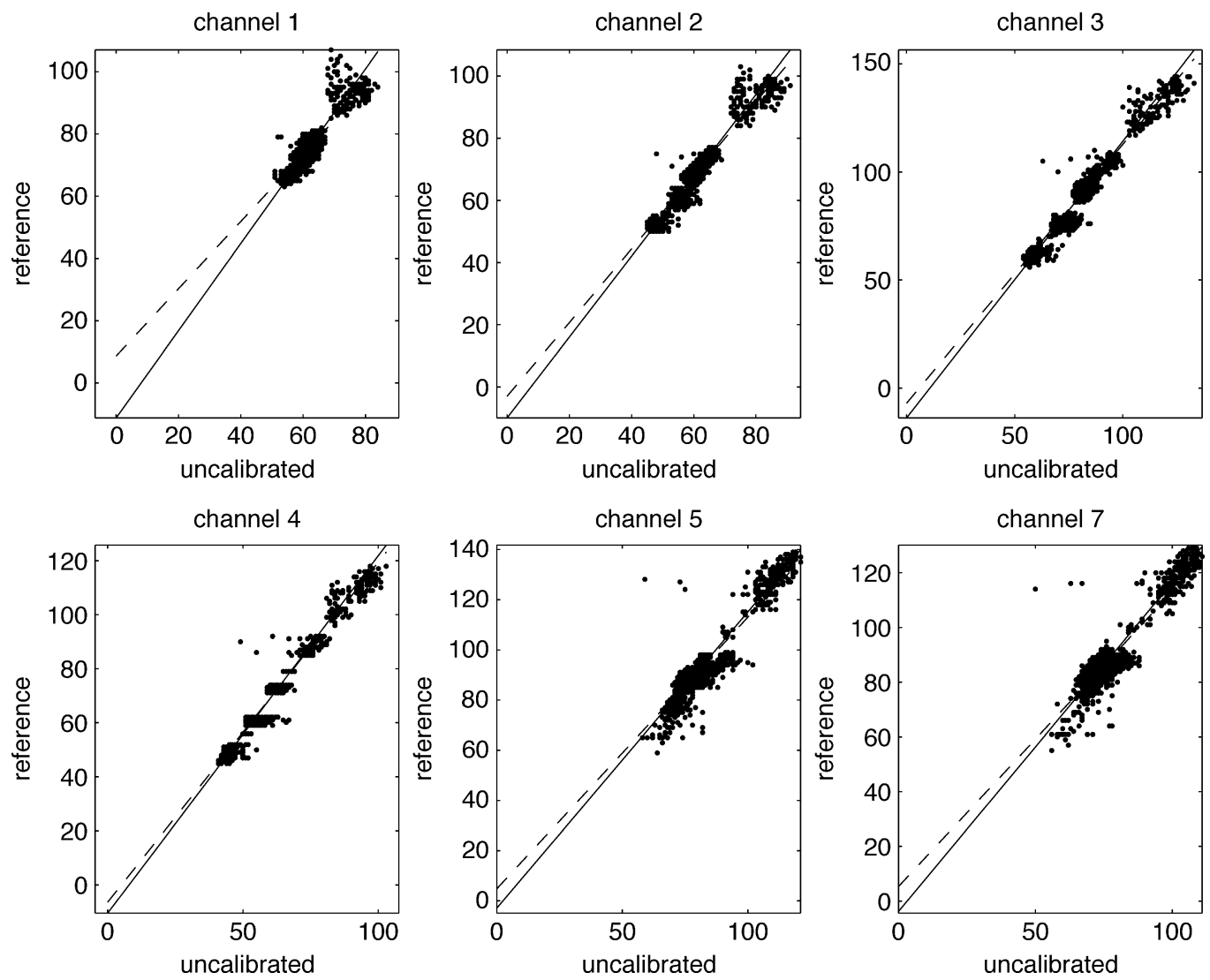

Fig. 4. Regression of the 1999 Morocco reference scene on the 2000 target (uncalibrated) scene using manually selected training pixels. Solid line: orthogonal regression; dashed line: ordinary least squares regression. 
Table 3

As in Table 2, for orthogonal regression

\begin{tabular}{lllllll}
\hline Band & \multicolumn{1}{l}{} & $\hat{\sigma}_{\alpha}$ & $\hat{\beta}$ & $\hat{\sigma}_{\beta}$ & $r$ & RMSE \\
\hline 1 & -11.22 & 0.72 & 1.400 & 0.011 & 0.818 & 1.273 \\
2 & -9.94 & 0.37 & 1.300 & 0.006 & 0.928 & 1.157 \\
3 & -13.79 & 0.41 & 1.280 & 0.005 & 0.947 & 1.734 \\
4 & -10.41 & 0.28 & 1.322 & 0.004 & 0.961 & 1.237 \\
5 & -2.95 & 0.44 & 1.180 & 0.005 & 0.927 & 1.916 \\
7 & -3.80 & 0.47 & 1.202 & 0.006 & 0.910 & 1.894 \\
\hline
\end{tabular}

comparison of the MAD procedure with normalization based on invariant features. The areas were selected on the basis of availability of ground reference data on features of constant reflectance. The dimensions of the scenes were $729 \times 754$ pixels. The Nevada data consisted of one Landsat- 4 TM and five Landsat-5 TM scenes taken at approximately monthly intervals in the second half of 1991. A region of interest $(1000 \times 1000$ pixels $)$ was chosen having some agricultural activity (pivot irrigation) and significant cloud cover at the time used as normalization reference, see Fig. 2. The Kenya data consisted of two SPOT HRV images recorded in 1987 and 1989 over an agricultural region near Thika just north of Nairobi, Fig. 3. The size of scenes was $512 \times 512$ pixels. These data were chosen to illustrate radiometric normalization in a non-arid region.

The Morocco and Nevada scenes were registered to one another by applying an automatic contour matching algorithm due to $\mathrm{Li}$ et al. (1995) and using second-order polynomial, nearest-neighbor resampling. The RMS errors were less than 0.5 pixel. The Kenya data were geocoded to a common reference map with similar accuracy.

\subsection{Morocco}

As mentioned above, the Morocco scenes, for which ground reference data were available, were used to compare the MAD procedure with normalization based on manual selection of invariant features; see, e.g. Schott et al. (1988). The features were chosen from dark, bright and medium reflectance surfaces representative of the surface variability, see Table 1.

In their original paper on "pseudo-invariant features" (PIFs), Schott et al. (1988) do not use ordinary linear

Table 4

Comparison of mean intensities of hold-out test pixels for the 2000 Morocco scene before and after normalization to the 1999 scene with ordinary least squares regression, with paired $t$-tests for equal means

\begin{tabular}{|c|c|c|c|c|c|c|}
\hline TM band & 1 & 2 & 3 & 4 & 5 & 7 \\
\hline Uncorrected(2000) & 62.080 & 59.898 & 81.975 & 62.612 & 77.989 & 72.898 \\
\hline Normalized(2000) & 75.720 & 67.969 & 91.143 & 72.400 & 89.117 & 83.820 \\
\hline Reference(1999) & 75.650 & 67.969 & 91.115 & 72.455 & 89.114 & 83.771 \\
\hline Difference & -0.069 & 0.000 & -0.027 & 0.055 & -0.003 & -0.049 \\
\hline$t$ & -2.207 & -0.001 & -0.589 & 1.668 & -0.069 & -1.062 \\
\hline$p$ & 0.027 & 0.998 & 0.555 & 0.095 & 0.944 & 0.287 \\
\hline
\end{tabular}

Table 5

Comparison of variances of hold-out test pixels for the 2000 Morocco scene before and after normalization to the 1999 scene with ordinary least squares regression, with $F$-tests for equal variances

\begin{tabular}{lccllll}
\hline TM band & 1 & 2 & 3 & 4 & 5 & 7 \\
\hline Uncorrected(2000) & 6.96 & 14.48 & 44.93 & 29.60 & 40.692 & 31.70 \\
Normalized(2000) & 8.14 & 20.34 & 64.52 & 46.85 & 47.60 & 36.77 \\
Reference(1999) & 10.88 & 22.09 & 68.98 & 49.16 & 54.16 & 43.27 \\
$F$ & 1.336 & 1.086 & 1.069 & 1.049 & 1.138 & 1.177 \\
$p$ & 0.000 & 0.013 & 0.0443 & 0.147 & 0.000 & 0.000 \\
\hline
\end{tabular}

regression, but rather assume a direct (error-free) linear relation between digital numbers recorded from man-made features at two times. Since imagery is always subject to stochastic measurement error, we prefer to use regression methods which allow for this error. Fig. 4 shows the orthogonal regressions (solid lines) for normalization of the two Morocco images, based on 2/3 of the no-change pixels (referred to henceforth as "training pixels") determined from the invariant features. For comparison, the results of ordinary least squares regression are also given (dashed lines). Note that orthogonal regression leads to a consistently higher slope and correspondingly smaller intercept than ordinary regression. The fitted intercepts $(\hat{\alpha})$ and slopes $(\hat{\beta})$ for ordinary regression are shown in Table 2 for the 7200 training pixels, those for orthogonal regression in Table 3. Tables 4 and 5 show, respectively, the means and variances of the 1999 scene before and after normalization to the 2000 scene using the ordinary least squares regression line. They were determined with the 3600 holdout test pixels. Tables 6 and 7 show similar results after normalization using the orthogonal regression lines.

In contrast with the manually selected data, Fig. 5 displays the orthogonal and ordinary least squares regressions for normalization of the two Morocco images based on 11260 no-change training pixels derived from the MAD procedure. Tables $8-13$ give the corresponding information on regression statistics and on the comparisons of means and variances with 5630 test pixels.

Comparing Tables 4 and 6 , we see that the paired $t$-tests for equal mean values of the individual bands after the manual normalization are better (the differences and $t$-values are closer to zero and the $p$-values are higher) for OLS regression for all bands except TM7. The $p$-value is the probability of finding a larger value of $|t|$. We also see that for all bands except TM1 for both OLS and orthogonal regression, none of the $p$-values are below 5\%. This means

Table 6

As in Table 4, for orthogonal regression

\begin{tabular}{lrrrrrr}
\hline TM band & 1 & \multicolumn{1}{l}{2} & \multicolumn{1}{l}{4} & 4 & \multicolumn{1}{l}{5} & \multicolumn{1}{l}{7} \\
\hline Uncorrected(2000) & 62.08 & 59.90 & 81.98 & 62.61 & 77.99 & 72.90 \\
Normalized(2000) & 75.73 & 67.97 & 91.15 & 72.40 & 89.11 & 83.81 \\
Reference(1999) & 75.65 & 67.97 & 91.12 & 72.46 & 89.11 & 83.77 \\
Difference & -0.084 & 0.000 & -0.030 & 0.058 & 0.005 & -0.044 \\
$t$ & -2.367 & 0.012 & -0.635 & 1.694 & 0.103 & -0.915 \\
$p$ & 0.018 & 0.991 & 0.525 & 0.090 & 0.918 & 0.360 \\
\hline
\end{tabular}


Table 7

As in Table 5, for orthogonal regression

\begin{tabular}{lcccccc}
\hline TM band & 1 & 2 & 3 & 4 & 5 & 7 \\
\hline Uncorrected(2000) & 6.97 & 14.49 & 44.93 & 29.60 & 40.69 & 31.70 \\
Normalized(2000) & 13.67 & 24.51 & 73.63 & 51.78 & 56.70 & 45.80 \\
Reference(1999) & 10.88 & 22.09 & 68.98 & 49.16 & 54.16 & 43.27 \\
$F$ & 0.796 & 0.901 & 0.937 & 0.949 & 0.955 & 0.945 \\
$p$ & 0.000 & 0.002 & 0.050 & 0.118 & 0.167 & 0.0868 \\
\hline
\end{tabular}

that we can assume that the band-wise mean values are equal after normalization except for TM1. A $T^{2}$-test for equality of the mean vectors of all bands after normalization does not show significant equality. The $T^{2}$-value is lower (19.865 vs. 21.793$)$ and the significance level is higher, i.e., better $(0.0030$ vs. 0.0014$)$ for OLS regression.

Comparing Tables 5 and 7, we see that the band-wise variances are quite different after normalization for both OLS and orthogonal regression. The $F$-values are the ratios between the variances of the reference data and the normalized data. These values should be close to one. The significance levels show that we can assume equal variances for TM4 with OLS and for TM3, TM4, TM5 and TM7 with orthogonal regression since these are all higher than $5 \%$.

Comparing Tables 9 and 12, we see that the paired $t$-tests for equal mean values of the individual bands after the
Table 8

Ordinary least squares regression on training MAD pixels for the Morocco scenes

\begin{tabular}{lllllll}
\hline Band & $\hat{\alpha}$ & $\hat{\sigma}_{\alpha}$ & $\hat{\beta}$ & $\hat{\sigma}_{\beta}$ & $r$ & RMSE \\
\hline 1 & -1.56 & 0.19 & 1.230 & 0.003 & 0.966 & 1.074 \\
2 & -4.68 & 0.13 & 1.191 & 0.002 & 0.978 & 1.372 \\
3 & -8.88 & 0.12 & 1.194 & 0.001 & 0.983 & 2.109 \\
4 & -8.31 & 0.10 & 1.265 & 0.002 & 0.987 & 1.546 \\
5 & -2.22 & 0.13 & 1.148 & 0.001 & 0.981 & 2.244 \\
7 & -1.33 & 0.14 & 1.146 & 0.002 & 0.976 & 1.983 \\
\hline
\end{tabular}

MAD-based normalization are better (the differences and $t$ values are closer to zero and the $p$-values are higher) for OLS regression for all bands. We also see that for all bands for both OLS and orthogonal regression, none of the $p$ values are below $5 \%$. This means that we can assume that the band-wise mean values are equal after normalization. Also the $T^{2}$-test for equality of the mean vectors of all bands after normalization shows significant equality. The $T^{2}$-value is lower (5.777 vs. 6.063) and significance level is higher, i.e., better (0.4493 vs. 0.4169$)$ for orthogonal regression.

In Tables 10 and 13, the $F$-tests for equal variances show that we cannot reject the hypothesis of equal variances for any band with orthogonal regression whereas we must reject
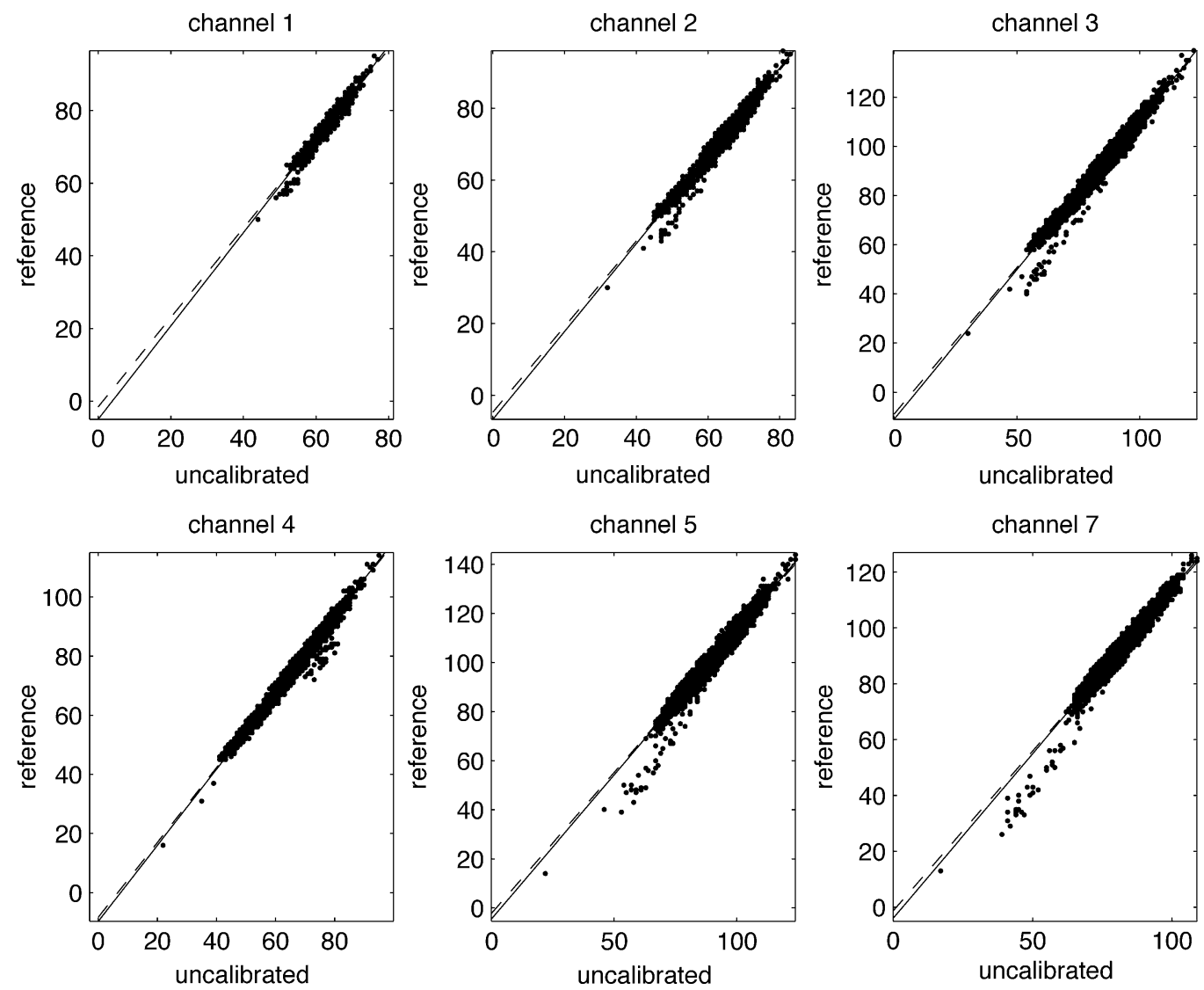

Fig. 5. Regression of the 1999 Morocco reference scene on the 2000 target (uncalibrated) scene using the MAD training pixels. Solid line: orthogonal regression; dashed line: ordinary least squares regression. 
Table 9

Comparison of mean intensities of hold-out test MAD pixels for the 2000 Morocco scene before and after normalization to the 1999 scene with ordinary least squares regression, with paired $t$-tests for equal means

\begin{tabular}{|c|c|c|c|c|c|c|}
\hline TM band & 1 & 2 & 3 & 4 & 5 & 7 \\
\hline Uncorrected(2000) & 62.734 & 61.544 & 83.894 & 64.573 & 88.128 & 80.094 \\
\hline Normalized(2000) & 75.577 & 68.621 & 91.319 & 73.345 & 98.936 & 90.441 \\
\hline Reference(1999) & 75.576 & 68.595 & 91.279 & 73.323 & 98.905 & 90.414 \\
\hline Difference & -0.001 & -0.026 & -0.039 & -0.022 & -0.032 & -0.027 \\
\hline$t$ & -0.059 & -1.416 & -1.390 & -1.079 & -1.052 & -1.020 \\
\hline$p$ & 0.953 & 0.157 & 0.165 & 0.280 & 0.293 & 0.308 \\
\hline
\end{tabular}

the hypothesis of equal variances for TM1 and TM7 for OLS regression.

Tables 2, 3, 8 and 11 show that the RMS errors are lower for MAD-based normalization and for orthogonal regression. This is true for all bands.

Finally, the plots in Figs. 4 and 5 clearly show a lot more scatter in the no-change pixels for the manual method corresponding to lower correlations as seen in Tables 2 (or 3 ) and 8 (or 11).

In spite of the better OLS fit for the means, all the above shows that in this case the automatic MAD-based normalization outperforms the manual normalization and that orthogonal regression is to be preferred over the OLS regression normally applied to normalization.

\subsection{Nevada}

Five of the Nevada images (August through December, 1991) were normalized to the July, 1991 image with the MAD procedure using orthogonal regression as described above. Fig. 6 displays the reference image (lower center) and of the December, 1991 target image before (upper left) and after normalization (upper right). The main spectral differences prior to normalization are due to Sun elevation, circular pivot plantations and clouds. Normalization to the July image as reference results in a qualitatively similar image for December. Since the clouds and irrigation pivots represent real changes, they have no influence on the calibration. The only other subjectively evident differences after normalization are the longer shadows in the December scene and some bidirectional reflectance effects in the mountainous areas.

For radiometric normalization over arid areas, both atmospheric differences and actual changes in surface re-

Table 10

Comparison of variances of hold-out test MAD pixels for the 2000 Morocco scene before and after normalization to the 1999 scene with ordinary least squares regression, with $F$-tests for equal variances

\begin{tabular}{|c|c|c|c|c|c|c|}
\hline TM band & 1 & 2 & 3 & 4 & 5 & 7 \\
\hline Uncorrected(2000) & 10.58 & 28.71 & 86.99 & 54.45 & 95.67 & 59.79 \\
\hline Normalized(2000) & 15.99 & 40.72 & 124.11 & 87.06 & 126.05 & 78.50 \\
\hline Reference(1999) & 16.92 & 42.43 & 128.44 & 89.26 & 131.27 & 82.86 \\
\hline$F$ & 1.058 & 1.042 & 1.035 & 1.025 & 1.041 & 1.056 \\
\hline$p$ & 0.035 & 0.121 & 0.197 & 0.348 & 0.126 & 0.042 \\
\hline
\end{tabular}

Table 11

As in Table 8, for orthogonal regression

\begin{tabular}{lrlllll}
\hline Band & \multicolumn{1}{l}{$\alpha$} & $\hat{\sigma}_{\alpha}$ & $\hat{\beta}$ & $\hat{\sigma}_{\beta}$ & $r$ & RMSE \\
\hline 1 & -4.96 & 0.20 & 1.284 & 0.003 & 0.966 & 0.670 \\
2 & -6.66 & 0.15 & 1.223 & 0.002 & 0.978 & 0.875 \\
3 & -10.98 & 0.18 & 1.219 & 0.002 & 0.983 & 1.346 \\
4 & -9.65 & 0.13 & 1.285 & 0.002 & 0.987 & 0.954 \\
5 & -4.53 & 0.20 & 1.174 & 0.002 & 0.981 & 1.465 \\
7 & -3.95 & 0.20 & 1.179 & 0.002 & 0.976 & 1.293 \\
\hline
\end{tabular}

flectance are likely to be small. Fig. 7 displays the overall mean pixel intensities in the six Landsat TM images before and after normalization to the July image. The intensities have been averaged over all six non-thermal bands. The means were calculated using the $33 \%$ holdout test pixels. Also shown in the figure are the unnormalized mean intensities multiplied by the factor

$\frac{d_{i}^{2}}{\cos \theta_{i}} \cdot \frac{\cos \theta_{1}}{d_{1}^{2}}, \quad i=1 \ldots 6$,

where $\theta_{i}$ is the Sun zenith angle and $d_{i}$ is the Earth-Sun distance for each of the six acquisition dates. Since the sensor gains and offsets were constant over the acquisition period, this is equivalent to a normalization without atmospheric correction. Therefore, the variations may be attributed to differences in atmospheric absorption and scattering.

\subsection{Kenya}

The Kenya data are from an agricultural region near Thika just north of Nairobi and were used to test the MAD normalization based on both OLS and orthogonal regression on data from a non-arid region. The images cover the town of Thika, large pineapple fields to the north and small coffee fields to the northwest of Thika.

Results for the test pixels (not shown) are similar to those of the data from arid regions: although we see more scatter and therefore less correlation (especially for band 3 ) than in the cases with arid data, both OLS and orthogonal regression give normalized data with the same mean as the reference data, OLS gives better significance. OLS regression gives significantly different variances whereas orthogonal regression gives equal variances. Also the RMSEs are smaller for orthogonal regression.

Fig. 8 shows the cumulative distribution functions for the three bands before and after MAD-based normalization with

Table 12

As in Table 9, for orthogonal regression

\begin{tabular}{lrrrrrr}
\hline TM band & 1 & 2 & 3 & 4 & 5 & \multicolumn{1}{l}{7} \\
\hline Uncorrected(2000) & 62.734 & 61.544 & 83.894 & 64.573 & 88.128 & 80.094 \\
Normalized(2000) & 75.580 & 68.625 & 91.324 & 73.349 & 98.943 & 90.447 \\
Reference(1999) & 75.576 & 68.595 & 91.279 & 73.323 & 98.905 & 90.414 \\
Difference & -0.004 & -0.030 & -0.044 & -0.026 & -0.039 & -0.033 \\
$t$ & -0.310 & -1.625 & -1.554 & -1.248 & -1.279 & -1.236 \\
$p$ & 0.757 & 0.104 & 0.120 & 0.212 & 0.201 & 0.217 \\
\hline
\end{tabular}


Table 13

As in Table 10, for orthogonal regression

\begin{tabular}{lllrrrr}
\hline TM band & 1 & \multicolumn{1}{l}{2} & \multicolumn{1}{l}{4} & \multicolumn{1}{l}{5} & \multicolumn{1}{l}{7} \\
\hline Uncorrected(2000) & 10.58 & 28.71 & 86.99 & 54.45 & 95.67 & 59.79 \\
Normalized(2000) & 17.44 & 42.96 & 129.37 & 89.96 & 131.89 & 83.06 \\
Reference(1999) & 16.92 & 42.43 & 128.44 & 89.26 & 131.27 & 82.86 \\
$F$ & 0.970 & 0.987 & 0.993 & 0.992 & 0.995 & 0.997 \\
$p$ & 0.254 & 0.644 & 0.784 & 0.766 & 0.858 & 0.927 \\
\hline
\end{tabular}

orthogonal regression: a visually pleasing fit has been obtained.

\section{Conclusions}

The procedure for radiometric normalization suggested here is automatic, very fast and requires, apart from the chi-

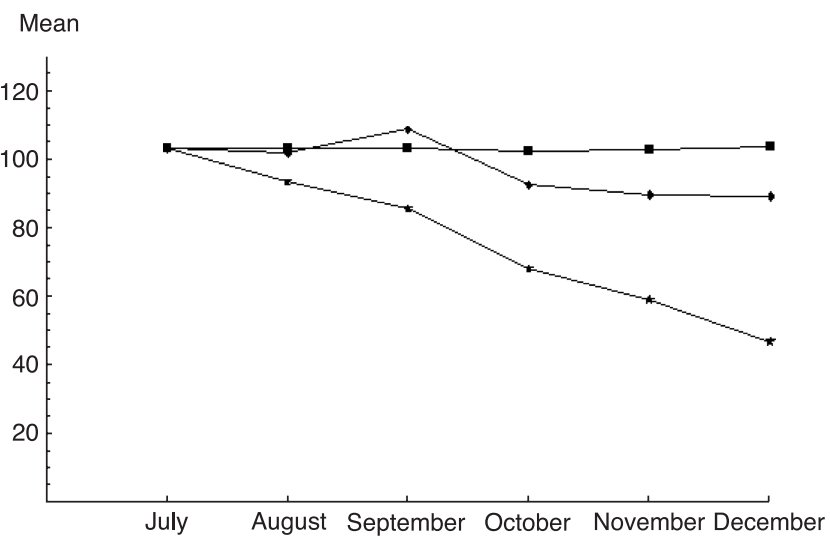

Fig. 7. Unnormalized (stars) and normalized (boxes) mean pixel intensities (in digital number units) for six Landsat TM images over Nevada from July to December, 1991. The July image was taken as reference. The diamonds are the unnormalized mean values corrected for Sun elevation and EarthSun distance (see text).

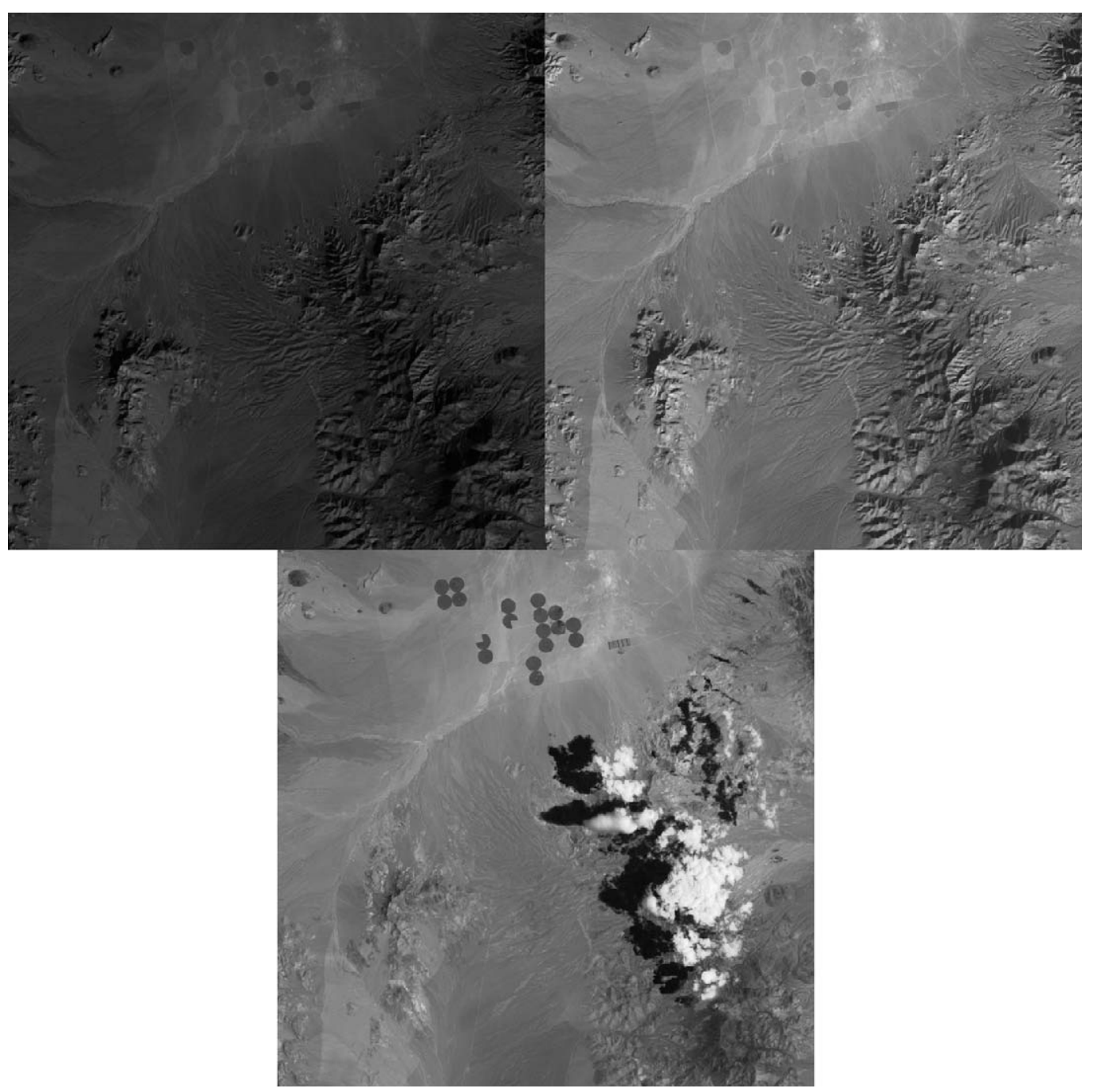

Fig. 6. Radiometric normalization of the Nevada scene. Top left: the uncorrected December, 1991 image; top right: the December scene after normalization; bottom middle: the July, 1991 reference scene. 

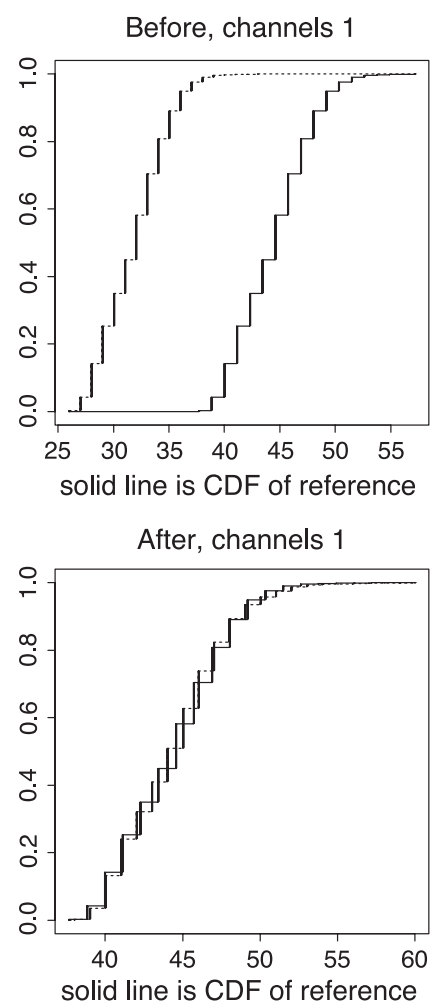

Before, channels 2

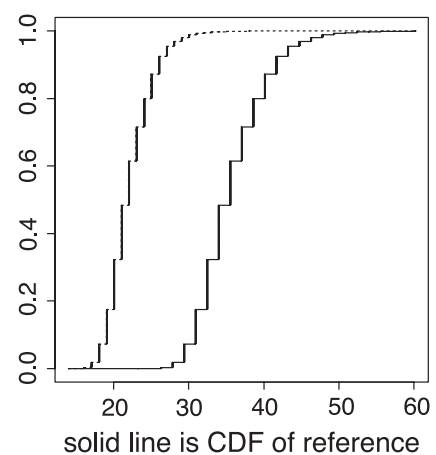

After, channels 2

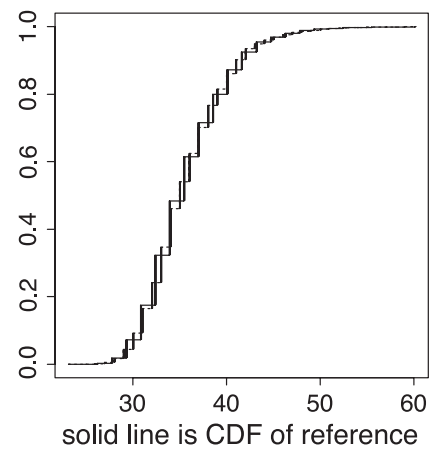

Before, channels 3

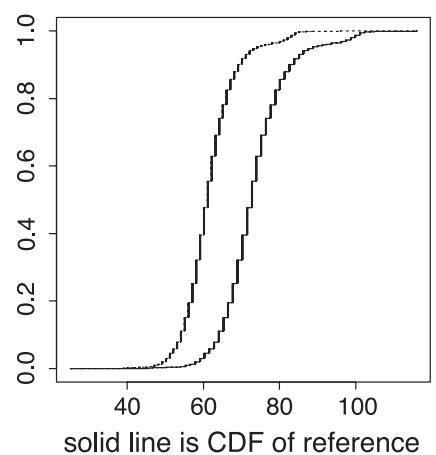

After, channels 3

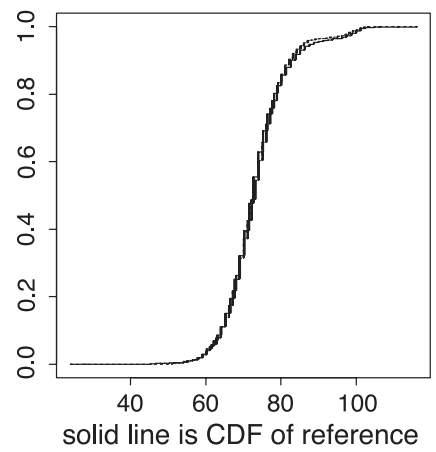

Fig. 8. Cumulative distribution functions for SPOT HRV bands before and after MAD-based normalization with orthogonal regression.

square percentile, no externally adjustable parameters such as decision thresholds or subjective criteria for defining PIF masks; everything else is entirely determined by the image data themselves. The method yields results which compare favorably to those obtained by the more laborious manual choice of time-invariant features in the images involved. On the whole, orthogonal regression using the no-change pixels is to be preferred to ordinary least squares regression. As the no-change pixels are actually selected for each image on the basis of multispectral change detection relative to the reference image, the method automatically avoids interference due to cloud cover, or indeed due to any other kind of reflectance changes that might occur.

In a recent proposal by $\mathrm{Du}$ et al. (2002), pseudoinvariant pixels are also selected using statistical properties rather than physical characteristics of reflecting surfaces. Their selection of suitable pixels for normalization is based on a bitemporal principal component transformation. Because of the presence of change pixels in the transformation, the principal axis must be recalculated after setting of rejection thresholds. Since the principal component transformation, unlike the MAD transformation, is not scale invariant, the method proposed here would appear to be better and more natural. Conservation of radiometric resolution after normalization, an aspect emphasized in Du et al. (2002), can of course be achieved similarly with the MAD method.

Finally, as an example of the application of relative radiometric normalization with MAD, Figs. 9 and 10 show

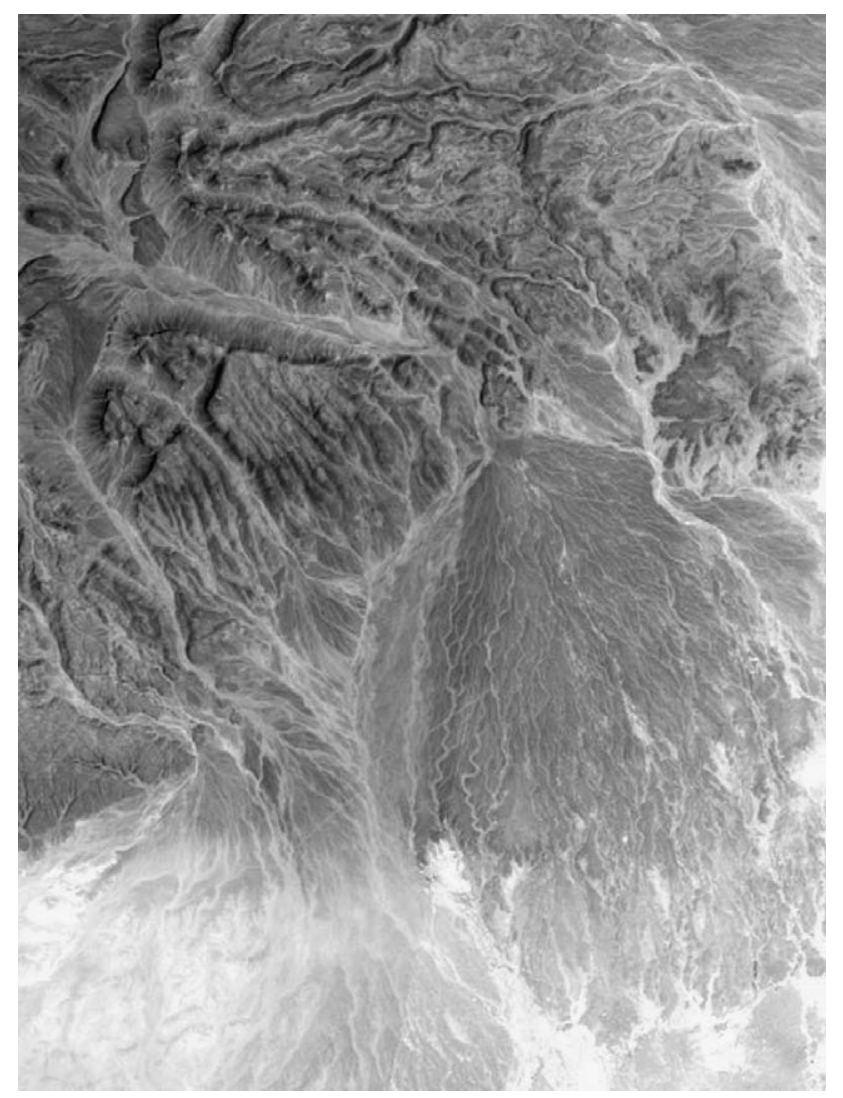

Fig. 9. Mosaic of two Landsat ETM+ scenes from May 2 and May 27, 2000 without radiometric normalization. 


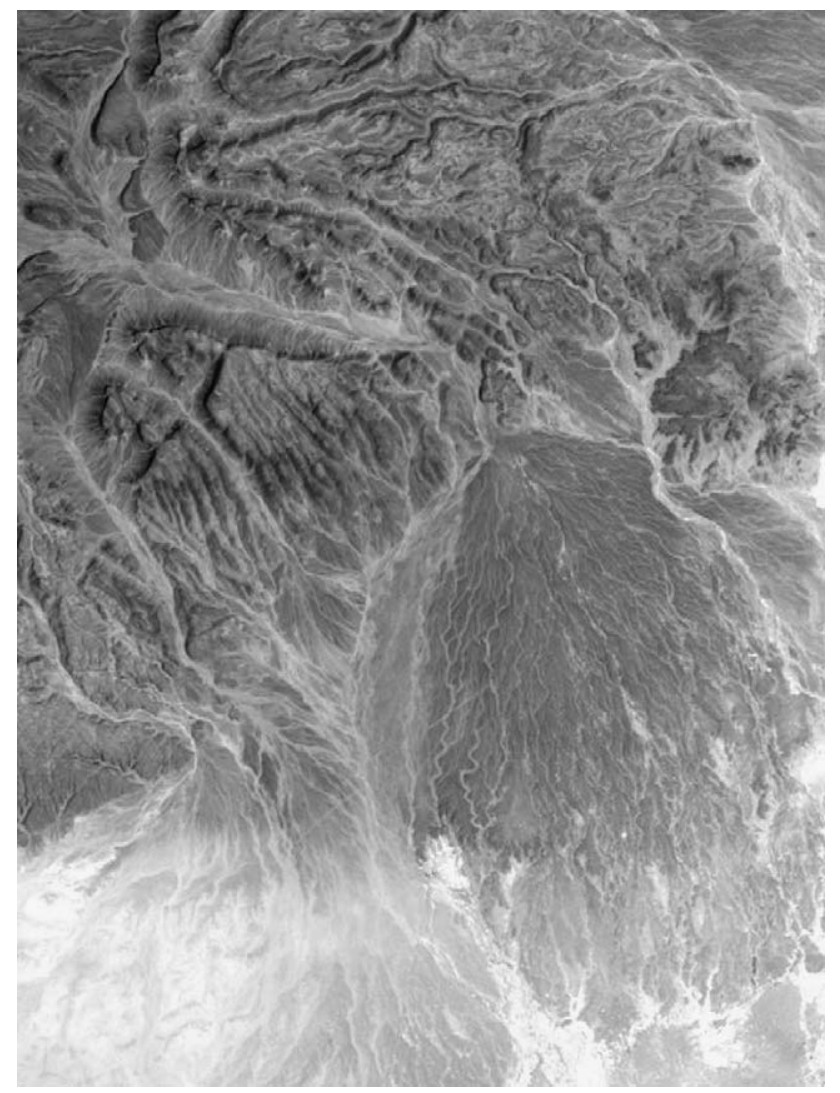

Fig. 10. As in Fig. 9, with radiometric normalization using the MAD procedure and orthogonal regression.

a part of the intersection area of a mosaic of Landsat ETM+ scenes over south Morocco on adjacent paths dating from May 2, 2000 and May 25, 2000. Fig. 9 is without, Fig. 10 with radiometric normalization. For Fig. 10 , a subset of the overlap area of the images was used to calculate the regression parameters. The true changes in the surface reflectance, still apparent in the figure after normalization, are the result of rainfall prior to the acquisition of the second scene, as is the difference in the water level in the river.

\section{Acknowledgements}

AAN thanks Dr. Poul Thyregod, IMM, Technical University of Denmark, for many good discussions on normalization and calibration.

\section{Appendix A}

Some readers may not be familiar with the two types of regression analysis applied in this paper. We therefore give a very brief account of some of the more important characteristics of the two.

\section{A.1. Ordinary least squares regression}

In the model for ordinary least squares (OLS) regression

$y_{i}=\alpha+\beta x_{i}+\gamma_{i}, \quad i=1 \ldots n$

where $x$ is considered as an independent (fixed, deterministic) predictor and $y$ is considered as a dependent (random, stochastic) response, the $x$ 's are assumed to be uncertainty- or error-free. (This usage of the terms dependent and independent is different from the usual probabilistic meaning.) $n$ is the number of observations and $\gamma$ is a white, Gaussian noise term with mean zero and variance $\sigma^{2}$, white meaning that $\gamma_{i}$ and $\gamma_{j}$ are stochastically independent if $i \neq j$.

In this model, the estimator for $\beta$ is (see any good textbook on statistics), for example (Rice, 1995)

$\hat{\beta}=\frac{s_{x y}}{s_{x x}^{2}}$

where

$s_{x y}=\frac{1}{n} \sum_{i=1}^{n}\left(x_{i}-\bar{x}\right)\left(y_{i}-\bar{y}\right)$,

$s_{x x}^{2}=\frac{1}{n} \sum_{i=1}^{n}\left(x_{i}-\bar{x}\right)^{2}$

with $n \bar{x}=\sum_{i=1}^{n} x_{i}$ and $n \bar{y}=\sum_{i=1}^{n} y_{i}$. The estimator for $\alpha$ is

$\hat{\alpha}=\bar{y}-\hat{\beta} \bar{x}$.

The variance/covariance matrix (also known as the dispersion matrix) of the vector $[\hat{\alpha} \hat{\beta}]^{T}$ is

$\frac{\sigma^{2}}{n \sum x_{i}^{2}-\left(\sum x_{i}\right)^{2}}\left[\begin{array}{cc}\sum x_{i}^{2} & -\sum x_{i} \\ -\sum x_{i} & n\end{array}\right]$

where $\sigma^{2}$ can be replaced by

$\hat{\sigma}^{2}=\frac{1}{n-2} \sum_{i=1}^{n} \hat{\gamma}_{i}^{2}$

with $\hat{\gamma}_{i}=y_{i}-\hat{\alpha}-\hat{\beta} x_{i}$. The root-mean-squared error (RMSE) is $\hat{\sigma}$.

The standard errors of $\hat{\alpha}$ and $\hat{\beta}$ are the square roots of the diagonal elements of the above dispersion matrix. The test statistics for $\hat{\alpha}$ and $\hat{\beta}$ being significantly different from zero are the estimates divided by the standard errors.

\section{A.2. Orthogonal regression}

In the model for ordinary least squares regression the $x$ 's are assumed to be error-free. In the calibration case where it is arbitrary what we call the reference variable and what we 
call the uncalibrated variable to be normalized, we should allow for error in both $x$ and $y$. If we impose the model (we reuse the symbols $\hat{\alpha}$ and $\hat{\beta}$, later also $\sigma$ )

$y_{i}-\epsilon_{i}=\alpha+\beta\left(x_{i}-\delta_{i}\right), \quad i=1 \ldots n$

with $\epsilon$ and $\delta$ as uncorrelated, white, Gaussian noise terms with mean zero and equal variances $\sigma^{2}$, we get for the estimator of $\beta$ (Kendall \& Stuart, 1979),

$\hat{\beta}=\frac{\left(s_{y y}^{2}-s_{x x}^{2}\right)+\sqrt{\left(s_{y y}^{2}-s_{x x}^{2}\right)^{2}+4 s_{x y}^{2}}}{2 s_{x y}}$

with

$s_{y y}^{2}=\frac{1}{n} \sum_{i=1}^{n}\left(y_{i}-\bar{y}\right)^{2}$

and the remaining quantities defined in the section immediately above. The model in Eq. (14) is often referred to as a linear functional relationship in the literature.The estimator for $\alpha$ is

$\hat{\alpha}=\bar{y}-\hat{\beta} \bar{x}$.

According to (Bilbo, 1989; Patefield, 1977), we get for the dispersion matrix of the vector $[\hat{\alpha} \hat{\beta}]^{T}$

$\frac{\sigma^{2} \hat{\beta}\left(1+\hat{\beta}^{2}\right)}{n s_{x y}}\left[\begin{array}{cc}\bar{x}^{2}(1+\hat{\tau})+s_{x y} / \hat{\beta} & -\bar{x}(1+\hat{\tau}) \\ -\hat{x}(1+\hat{\tau}) & 1+\hat{\tau}\end{array}\right]$

with

$\hat{\tau}=\frac{\sigma^{2} \hat{\beta}}{\left(1+\hat{\beta}^{2}\right) s_{x y}}$

and where $\sigma^{2}$ can be replaced by

$\hat{\sigma}^{2}=\frac{n}{(n-2)\left(1+\hat{\beta}^{2}\right)}\left(s_{y y}^{2}-2 \hat{\beta} s_{x y}+\hat{\beta}^{2} s_{x x}^{2}\right)$,

It can be shown that estimators of $\alpha$ and $\beta$ can be calculated by means of the elements in the eigenvector corresponding to the smallest eigenvalue of the dispersion matrix of the $n$ by two data matrix with a vector of the $x$ 's in the first column and a vector of the $y$ 's in the second column (Kendall \& Stuart, 1979). This can be used to perform orthogonal regression in higher dimensions, i.e., when we have, for example, more $x$ variables than the one variable we have in our case.

Software packages to perform ordinary least squares regression (LAPACK) and orthogonal regression (ODRPACK) can be found on the Internet.

\section{References}

Bilbo, C. M. (1989). Statistisk analyse af relationer mellem alternative antistoftracere. Master's thesis, Informatics and Mathematical Modeling, Technical University of Denmark, Lyngby, 1989. In Danish.

Du, Y., Teillet, P. M., \& Cihlar, J. (2002). Radiometric normalization of multitemporal high-resolution images with quality control for land cover change detection. Remote Sensing of Environment, 82, 123-134.

Furby, S. L., \& Campbell, N. A. (2001). Calibrating images from different dates to like-valuecounts. Remote Sensing of Environment, 77, $186-196$.

Hall, F. G., Strebel, D. E., Nickeson, J. E., \& Goetz, S. J. (1991). Radiometric rectification: Toward a common radiometric response among multidate, mutisensor images. Remote Sensing of Environment, 35, $11-27$.

Kendall, M., \& Stuart, A. (1979) (4th ed.). The advanced theory of statistics, vol. 2. London: Charles Griffen.

Li, H., Manjunath, B. S., \& Mitra, S. K. (1995). A contour-based approach to multisensor image registration. IEEE Transactions on Image Processing, 4(3), 320-334.

Moran, M. S., Jackson, R. D., Slater, P. N., \& Teillet, P. M. (1992). Evaluation of simplified procedures for retrieval of land surface reflectance factors from satellite sensor output. Remote Sensing of Environment, 41, $160-184$

Nielsen, A. A., Conradsen, K., \& Andersen, O. B. (2002). A change oriented extension of EOF analysis applied to the 1996-1997 AVHRR sea surface temperature data. Physics and Chemistry of the Earth, 27(32-34), 1379-1386.

Nielsen, A. A., Conradsen, K., \& Simpson, J. J. (1998). Multivariate alteration detection (MAD) and MAF post-processing in multispectral, bitemporal image data: New approaches to change detection studies. Remote Sensing of Environment, 64, 1-19.

Patefield, W. M. (1977). On the information matrix in the linear functional problem. Journal of the Royal Statistical Society Series C, 26, 69-70.

Rice, J. A. (1995). Mathematical statistics and data analysis (2nd ed.). Belmont, California: Duxbury Press/Wadsworth.

Schott, J. R., Salvaggio, C., \& Volchok, W. J. (1988). Radiometric scene normalization using pseudo-invariant features. Remote Sensing of Environment, 26, 1-16.

Yang, X., \& Lo, C. P. (2000). Relative radiometric normalization performance for change detection from multi-date satellite images. Photogrammetric Engineering and Remote Sensing, 66, 967-980. 